\title{
Verificação de usabilidade em software educativo: uma avaliação prospectiva em objetos de aprendizagem sobre genética
}

\author{
Usability check in educational software: a prospective assessment on \\ learning objects on genetics
}

\begin{abstract}
Verificación de la usabilidad en el software educativo: una evaluación prospectiva sobre los objetos del aprendizaje en genética
\end{abstract}

Gisele Patricia Scapini ${ }^{1}$; Vanessa Faria de Souza ${ }^{2}$; Mayara Faria de Souza ${ }^{3}$

\section{RESUMO}

Uma tendência é que a sala de aula se torne um ambiente cada vez mais dinâmico e interativo, fatores que podem motivar de forma abrangente os alunos e isso pode ser alcançado, por meio da aplicação de Objetos de Aprendizagem (OAs) digitais. Nesse sentido, o presente trabalho visou avaliar por prospecção, a usabilidade de dois OAs disponíveis no repositório NOAS (Núcleo de Desenvolvimento de Objetos de Aprendizagem Significativa), com temática vinculada a genética. Para isso, foi utilizado um questionário já consolidado chamado ErgoList, essa ferramenta é baseada nos critérios ergonômicos de Scapin e Bastien (1993) e foi desenvolvido pelo LabiUtil (laboratório de Utilizabilidade da Informática da Universidade Federal de Santa Catarina) (CYBIS, 1997; CYBIS et al., 2000; CYBIS et al., 2010), o qual foi aplicado a professores experientes e graduados em Ciências Biológicas. Os resultados demonstraram que alguns critérios de usabilidade não atenderam às expectativas dos docentes, porém isso não seria um empecilho na eficiência/eficácia da utilização dos OAs. De acordo com os professores, estes recursos seriam utilizados em suas aulas devido sobretudo a forma como o conteúdo de genética é abordado, que pode ser motivador aos alunos. Sendo assim, os OAs selecionados para esse estudo podem ser considerados recursos facilitadores do processo de ensino e aprendizagem de genética no Ensino Médio.

Palavras-chave: Objetos de aprendizagem; Avaliação de Usabilidade; Ensino Médio; Genética.

\begin{abstract}
One trend is that the classroom becomes an increasingly dynamic and interactive environment, factors that can motivate students in a comprehensive way, and this can be achieved through the application of digital Learning Objects (LOS). In this sense, the present work aimed to evaluate, by prospecting, the usability of two LOs available in the NOAS repository (Nucleus for the Development of Meaningful Learning Objects), with thematic linked to genetics. For this, an already consolidated questionnaire called ErgoList was used, this tool is based on the ergonomic criteria of Scapin and Bastien (1993) and was developed by LabiUtil (Computer Usability Laboratory of the Federal University of Santa Catarina) (CYBIS, 1997; CYBIS et al., 2000; CYBIS et al., 2010), which was applied to experienced teachers and graduates in Biological Sciences. The results showed that some usability criteria did not meet the expectations of teachers, but this would not be an obstacle to the efficiency/effectiveness of the use of LOs. According to the teachers, these resources would be used in their

\footnotetext{
${ }^{1}$ Mestra em Engenharia Florestal, graduada em Ciências Biológicas e Professora nas redes estadual, municipal e particular do município de Ibirubá/RS - Brasil. E-mail: gisele-pscapini@educar.rs.gov.br

2 Doutoranda no Programa de Pós-Graduação em Informática na Educação (PPGIE) da Universidade Federal do Rio Grande do Sul (UFRGS) e docente no Instituto Federal do Rio Grande do Sul (IFRS), Ibirubá/RS - Brasil. E-mail: vanessa.souza@ibiruba.ifrs.edu.br

3 Mestranda no Programa de Pós-Graduação em Ensino de Ciências Humanas, Sociais e da Natureza pela Universidade Federal Tecnológica do Paraná (UTFPR) e Professora da rede Municipal de Bandeirantes/PR Brasil. E-mail: mayarafariasouza7@gmail.com
} 
classes mainly due to the way in which the genetics content is approached, which can be motivating for students. Therefore, the LOs selected for this study can be considered resources that facilitate the teaching and learning process of genetics in high school.

Keywords: Learning Objects; Usability Assessment; High School; Genetics.

\section{RESUMEN}

Una tendencia es que el aula se convierta en un entorno cada vez más dinámico e interactivo, factores que pueden motivar a los estudiantes de manera integral, y esto se puede lograr mediante la aplicación de Objetos de Aprendizaje (OA) digitales. En este sentido, el presente trabajo tuvo como objetivo evaluar, mediante prospección, la usabilidad de dos OA disponibles en el repositorio NOAS (Núcleo de Desarrollo de Objetos de Aprendizaje Significativos), con temática vinculada a la genética. Para ello, se utilizó un cuestionario ya consolidado llamado ErgoList, esta herramienta está basada en los criterios ergonómicos de Scapin y Bastien (1993) y fue desarrollada por LabiUtil (Laboratorio de usabilidad informática de la Universidad Federal de Santa Catarina) (CYBIS, 1997; CYBIS et al., 2000; CYBIS et al., 2010), que se aplicó a profesores experimentados y licenciados en Ciencias Biológicas. Los resultados mostraron que algunos criterios de usabilidad no cumplían con las expectativas de los docentes, pero esto no sería un obstáculo para la eficiencia / efectividad del uso de los OA. Según los docentes, estos recursos se utilizarían en sus clases principalmente por la forma en que se abordan los contenidos de genética, lo que puede resultar motivador para los alumnos. Por tanto, los OA seleccionados para este estudio pueden considerarse recursos que facilitan el proceso de enseñanza $y$ aprendizaje de la genética en el bachillerato.

Palabras clave: Objetos de aprendizaje; Evaluación de Usabilidad; Escuela Secundaria; Genética.

\section{INTRODUÇÃO}

A sociedade atual convive diariamente com inovações tecnológicas, tais mudanças são constantes e com velocidade considerável e podem dar suporte a avanços e melhorias em diversas áreas. $\mathrm{O}$ maior exemplo de como as inovações tecnológicas podem apoiar, sobretudo na área educacional, foi a emergência do ensino remoto devido a pandemia da Covid 19. Nesse sentido, as tecnologias permitiram que os professores continuassem lecionando, mesmo que muitas vezes em condições adversas, mas mantendo um vínculo ativo com a sala de aula. $O$ desenvolvimento tecnológico alcançado no período de pandemia é uma construção que deve perdurar para além deste período.

A tecnologia da informação tem permeado o campo da educação em diversos níveis, mesmo antes da pandemia, o que resultava em um grande desafio para o professor mais adepto de recursos tradicionais. Todavia, a escola hoje pode ser considerada como um espaço para o desenvolvimento da aprendizagem de forma autônoma, e nesse sentido o uso da tecnologia não é mais questionado, mas sim estimulado, devido principalmente a abrangência e disponibilidade da internet e de tecnologias que apoiam o ensino e a aprendizagem. Sobretudo com a emergência do ensino remoto, não há mais que se questionar sobre a importância das tecnologias no âmbito educacional.

Neste contexto, os Objetos de Aprendizagem (OAs) podem desempenhar um papel importante no reforço do discurso educacional. Logo, inserir o uso dos OAs como forma de materializar o discurso do professor, e ainda proporcionar melhor contextualização do ensino se mostra bastante positivo, ainda mais quando o conteúdo abordado não é simples, como nas aulas de genética no Ensino Médio, tópico por vezes abstrato, embora muito presente no cotidiano dos estudantes e cobrado em avaliações como ENEM (Exame Nacional do Ensino Médio) e vestibulares. No entanto, como a maioria 
dos produtos no mercado, mesmo os de software, nem todos os OAs disponíveis são adequados e produtivos para o ensino e aprendizagem, e a consciência sobre como avaliar de forma adequada um software educacional, como um OA digital, é muito importante para os educadores.

Há várias formas e objetivos na avaliação de um software educativo, e uma das principais formas de se avaliar é a prospectiva, que se caracteriza principalmente pela participação do usuário que emite sua opinião com base na utilização do software. Este tipo de avaliação é baseado especialmente na aplicação de questionários (DAGNINO; DIAS, 2007). Quanto ao objetivo da avaliação, um dos principais requisitos a ser avaliado é o da usabilidade, que diz respeito a capacidade de um produto de software satisfazer as necessidades do usuário de forma simples e eficiente.

Diante deste contexto e da problemática apresentada, o principal objetivo deste estudo foi avaliar a usabilidade de dois OAs disponíveis no Núcleo de Desenvolvimento de Objetos de Aprendizagem Significativa (NOAS) ${ }^{4}$, ambos com temática vinculada a genética, verificando se estes estavam condizentes para utilização dos alunos. A avaliação realizada foi prospectiva, com a aplicação de um questionário já consolidado na área, o ErgoList. Esta ferramenta é considerada um tipo de checklist para avaliação de software intermediário, pois não é genérico, mas também não é específico para um único software, porém para uma classe de softwares. O ErgoList é baseado nos critérios ergonômicos de Scapin e Bastien (1993) e foi desenvolvido no LabiUtil laboratório de Utilizabilidade da Informática da Universidade Federal de Santa Catarina (CYBIS, 1997; CYBIS et al., 2000; CYBIS et al., 2010), este se concentra nos principais critérios de usabilidade que um software educativo deve possuir: (1) Compatibilidade, (2) Flexibilidade, (3) Legibilidade, (4) Controle do usuário, (5) Agrupamento/distinção por localização, (6) Significado dos códigos e denominações, (7) Presteza, (8) Ações Mínimas, (9) Consistência e (10) Densidade informacional. Como já salientado a avaliação prospectiva requer a participação de possíveis usuários do software, neste estudo representados por professores experientes e graduados em Ciências Biológicas.

Dessa forma, a principais contribuições pretendidas com esse estudo são: verificar se os OAs avaliados são condizentes quanto aos aspectos de usabilidade avaliados; explicitar de forma simples e didática como pode ser realizada a avaliação de usabilidade de um software educativo, como um Objeto de Aprendizagem; e esclarecer sobre a importância da avaliação em softwares educativos que priorizem um processo de ensino e aprendizagem de qualidade.

Para atendimento ao objetivo descrito, primeiramente foi realizada uma pesquisa bibliográfica, organizada em seções que aborda os seguintes temas: Seção 2 - Ensino de genética no Ensino Médio; Seção 3 - Informática na Educação; Seção 4 - Objetos de Aprendizagem, em que é também destacada a descrição dos OAs avaliados neste estudo; Seção 5 - Usabilidade em Ambientes Informatizados de Educação, em que também é abordada a perspectiva da avaliação de softwares educativos. Na sequência é descrita na Seção 6 a Metodologia empregada neste estudo; na Seção 7 são abordados os Resultados e Discussões; por fim, a Seção 8 traz as Considerações Finais deste estudo.

\footnotetext{
${ }^{4}$ NOAS - disponível em: https://www.noas.com.br/ensino-medio/biologia/genetica/
} 


\section{O ENSINO DE GENÉTICA NO ENSINO MÉDIO}

A Genética, além de ser uma área integradora da Biologia, é um dos ramos que mais tem apresentado mudanças nos últimos tempos, tanto nos seus aspectos tecnológicos como conceituais, sendo uma ciência fundamental e aplicada (JUSTINA, 2001). Moura (2013) define genética como:

[...] ciência da hereditariedade e o ramo da biologia que estuda os mecanismos de transmissão das características de uma espécie, passados de uma geração para outra, além das variações que ocorrem na transmissão das características e a importância delas na constituição dos organismos e na construção de tecnologias. (MOURA, 2013, p. 10).

Rocha (2013) menciona que muitos são os motivos que tornam o ensino de Genética indispensável na formação de qualquer indivíduo, pois a todo o momento existe a necessidade de tomadas de decisões que afetam a saúde e o bem-estar, bem como conhecimentos biológicos ajudam a tomar decisões mais adequadas no sentido de conservar a vida, fazendo as melhores escolhas. Apesar da importância que a Genética tem dentro da Biologia, estudos demonstram que os alunos do ensino médio consideram a Genética um tema bastante abstrato e difícil de aprender, sendo inúmeras as problemáticas enfrentadas pelos professores no processo de ensino e aprendizagem (SOUZA, 2015). Bonzanini e Bastos (2011) corroboram dizendo que isso ocorre também pela dificuldade encontrada pelos professores, pois tratam de assuntos relativamente novos, os quais na maioria das vezes não foram abordados durante o seu período de formação acadêmica.

Barni (2010) afirma em sua pesquisa que o ensino de diferentes áreas da ciência, inclusive a Genética, podem ficar limitadas ao uso do livro didático, não abordando os fatores históricos que levaram a construção daquele conhecimento. Silva, Fernandes e Sobreira (2018) também levam em consideração a carga horária e a correria que um professor de ensino médio é submetido, e muitas vezes esses não têm tempo suficiente para se atualizar, criar práticas novas de ensino e aulas mais dinâmicas e lúdicas.

\section{INFORMÁTICA NA EDUCAÇÃO}

A revolução da informática trouxe consigo inúmeros impactos que atingiram diversas áreas sociais e a educação não escapa dessa mudança. Cada vez mais a tecnologia se faz presente na escola e no aprendizado do aluno, seja pelo uso de equipamentos tecnológicos, seja por meio de projetos envolvendo educação e tecnologia (OLIVEIRA; MOURA; SOUSA, 2015). Para Valente e Almeida (1997) a história da informática na educação no Brasil nasceu no início dos anos 70 a partir de algumas experiências na Universidade Federal do Rio de Janeiro (UFRJ), Universidade Federal do Rio Grande do Sul (UFRGS) e Universidade Estadual de Campinas (UNICAMP). Nos anos 80 se estabeleceu, por meio de diversas atividades, estas permitiram que essa área hoje tenha uma identidade própria, raízes sólidas e relativa maturidade. Santos (2003) menciona projetos públicos como o Educom, Eureka e Gênese complementado por Souza (2013) que afirma que o setor público começou a investir na informática educacional, elaborando alguns projetos pioneiros que passaram a contribuir para o fortalecimento da informática nas escolas.

Por outro lado, as escolas particulares investiram na criação de disciplinas de informática, a partir do desenvolvimento de equipamentos de porte menor, os chamados computadores pessoais nas quais se ensinava a informática e não se ensinava com informática (TAVARES, 2002). Morellato (2006) contribui expondo que a informática na educação assume diversos significados dependendo da visão 
educacional e da condição pedagógica em que o computador é utilizado. Esses significados facilitam a construção do conhecimento, pois possibilitam a aplicação de diferentes metodologias e formas de conduzir o processo de ensino e aprendizagem (ADOLFO; MACHADO; WARPECHOWSKI, 2017).

\section{OBJETOS DE APRENDIZAGEM}

Com o advento da internet foi disponibilizado uma grande gama de materiais que podem ser utilizados para auxiliar no processo de ensino e aprendizagem. Os professores têm à sua disposição recursos de variados formatos e níveis de ensino para serem inseridos nos planos de aula (GUEDES; CASTRO FILHO, 2010), dentre eles os Objetos de Aprendizagem (OAs). Apesar de não haver um consenso sobre a definição referente aos objetos de aprendizagem, Wiley (2002) define OA como qualquer recurso digital que possa ser reutilizado para apoiar a aprendizagem. Nesse sentido, Tarouco (2014) determina que os OAs podem ser usados para o ensino de diversos conteúdos e revisão de conceitos, segundo a autora a flexibilidade e possibilidade de reutilização são as principais características de um $\mathrm{OA}$, estas facilitam a disseminação do conhecimento, assim como sua atualização.

Uma forma simples e rápida de ter acesso a diversos OAs é por meio dos repositórios digitais. Segundo Arellano (2008) os repositórios digitais constituem-se como sistemas de informação que servem para armazenar, preservar, organizar e difundir os resultados da produção intelectual de comunidades científicas e desse modo, em tais repositórios têm-se disponibilizados os OAs. Como exemplos de repositórios podem ser citados: o Banco Internacional de Objetos Educacionais, que possui objetos educacionais de acesso público, em vários formatos e para todos os níveis de ensino; o NOAS (Núcleo de Desenvolvimento de Objetos de Aprendizagem Significativa); e o RIVED (Rede Interativa Virtual de Educação); dentre outros.

Os OAs avaliados neste trabalho estão disponíveis no NOAS, este é um repositório que possui diversos objetos de aprendizagem com acesso gratuito e é mantido pela Campanha Nacional de Escolas da Comunidade (CNEC). Esse repositório disponibiliza diversos tipos de Objetos de Aprendizagem, principalmente no formato de jogos e simulações de fenômenos, para diversas faixas etárias, desde a educação infantil até o ensino superior, com plataforma acessível a professores e alunos. A escolha do NOAS, bem como dos objetos de aprendizagem se deu por suas características, as quais condizem com a proposta deste estudo. Em se tratando de genética, os OAs escolhidos foram os que mais se adequaram, no que se refere a elucidação de conteúdos abstratos, que dificultam a aprendizagem nas aulas sobre este conteúdo, também alvo dessa pesquisa. Os OAs que são avaliados neste trabalho são detalhados nas subseções em sequência.

\subsection{Montando Idiogramas}

Neste OA (indicado para o Ensino Médio) os alunos podem simular a montagem de idiogramas a partir de amostras de cariótipos humanos normais ou portadores de aneuploidias (alterações cromossômicas numéricas que se caracterizam pelo aumento ou diminuição de um tipo de cromossomo), assim este recurso pode auxiliar os discentes na revisão de conceitos genéticos e sua utilização é bastante intuitiva. Ao iniciar, o aluno receberá algumas instruções e depois conceitos relativos ao aplicativo; em seguida, em um laboratório virtual, poderá manipular amostras de sangue e soluções a fim de observar as reações; por fim, um idiograma será montado. O Objeto está 
disponível no repositório NOAS5 , é online, acessível para qualquer navegador, sua implementação foi baseada em Flash, responsável pela criação de animações interativas que funcionam embutidas em um navegador web.

\subsection{Síntese Proteica}

Este OA foi desenvolvido e disponibilizado pela Biblioteca Digital de Ciências da Unicamp e contém as opções: guia do professor, ajuda, sobre e sair. O conteúdo disponível nesse objeto é uma animação interativa da síntese proteica e tem como objetivo mostrar como ocorre tal processo nas células. Para isso, a barra de menu permite que o usuário navegue ao ponto do programa desejado, podendo ser: (1) Apresentação inicial, no qual são apresentados os objetivos e conteúdo do programa; (2) Introdução, em que é apresentado um texto de introdução ao tema síntese proteica; (3) Simulação, no qual pode-se simular a síntese de uma pequena proteína hipotética; (4) Animação, em que podese ver uma animação do processo de síntese proteica; (5) Sobre este programa, apresenta os créditos e outras informações relevantes do software; (6) Sair, opção para sair do programa. O OA está disponível no repositório $\mathrm{NOAS}^{6}$, é online, acessível para qualquer navegador e sua implementação foi baseada em Flash.

\section{USABILIDADE EM AMBIENTES INFORMATIZADOS DE EDUCAÇÃO}

A usabilidade é definida como a capacidade que um sistema interativo oferece a seu usuário, em um determinado contexto de operação, para a realização de tarefas, de maneira eficaz, eficiente e agradável (ISO 9241). A intuitividade, a facilidade e a eficiência de uso em um dispositivo informatizado contribuem para sua usabilidade, e ela visa a adaptação do trabalho ao ser humano, por meio de sistemas e dispositivos que estejam adaptados a maneira como o usuário pensa e trabalha. Além disso, a usabilidade mede a facilidade do usuário em completar objetivos específicos com eficácia, utilizando um produto projetado para proporcionar eficiência e satisfação ao usuário, em um contexto específico (ISO 9241).

A usabilidade é um aspecto importante na construção de sistemas de informação, websites, softwares educativos, dentre outros, visto que a primeira experiência do usuário é determinante para o seu retorno. A dificuldade de um usuário encontrar o que deseja, de navegar nas páginas e menus, ou perder tempo tentando entender uma interface, afugenta esse usuário que procurará opções mais intuitivas e agradáveis (NIELSEN; LORANGER, 2007). A usabilidade também é definida, ou medida para um contexto em que um sistema é operado. Assim, um sistema pode proporcionar boa usabilidade para um usuário experiente, mas péssima para novatos, ou vice e versa.

Pode ser fácil de operar uma vez apenas, mas difícil, se for utilizado no dia a dia. Pode dar prazer se acessado por conexões rápidas, mas causar ansiedade se acessado de casa. Maguire (2016) afirma sobre as inter-relações entre contexto de operação e usabilidade, a necessidade de especificar o contexto de uso para o qual uma interface está sendo concebida, e no qual ela será testada. O autor ainda salienta que a adaptabilidade é uma das boas qualidades de uma interface com o usuário. Essa característica permite que diferentes usuários, em diferentes estágios de competência, em diferentes

\footnotetext{
${ }^{5}$ Montando Idiogramas - http://www.noas.com.br/ensino-medio/biologia/genetica/montando-idiogramas/

${ }^{6}$ Síntese Proteica - http://www.noas.com.br/ensino-medio/biologia/genetica/sintese-proteica/
} 
tarefas e em diferentes ambientes físicos, tecnológicos e organizacionais, possam alcançar seus objetivos com eficácia, eficiência e satisfação.

\subsection{Perspectiva da avaliação: Objetivos de uma avaliação de usabilidade}

Avaliar um software educacional, como um OA, é uma etapa fundamental quando se visa alcançar um ensino qualificado. Carvalho (2005) defende a descoberta do usuário mediante a informação dada de maneira parcial, para que ele se sinta motivado a explorar cada vez mais informações novas que auxiliem no seu processo de cognição, ou seja, o objeto (software) deve se mostrar atraente e desafiador. Cybis et al. (2010) alertam sobre a importância de se ter uma interface agradável, priorizando a usabilidade e o alcance dos objetivos do usuário. Isso se torna crucial em softwares educacionais, pois quanto mais acessível de manusear, mais os alunos vão se interessar em descobrir os diferenciais que o programa pode the oferecer.

Nielsen (1993) também destaca a importância da usabilidade, ao subdividir o assunto em cinco critérios básicos a serem alcançados pelo software: (1) Intuitividade, o programa deve apresentar facilidade de uso; (2) Eficiência, o sistema deve ser eficiente em seu desempenho apresentando um alto nível de produtividade; (3) Memorização, suas telas devem apresentar facilidade de memorização; (4) Erro, a quantidade de erros apresentados pelo sistema deve ser o mais reduzido possível; e (5) Satisfação, o sistema deve agradar ao usuário, sejam eles iniciantes ou avançados. Com o intuito de garantir a qualidade, existem diversas ferramentas e metodologias que podem ser utilizadas para avaliar um software, cada uma delas com diferentes abordagens e facilidades. A avaliação de usabilidade de um sistema interativo tem como objetivos gerais verificar e validar: (1) a eficácia da interação humano-computador face a efetiva realização das tarefas por parte dos usuários; (2) a eficiência dessa interação, face os recursos empregados (tempo, quantidade de incidentes, passos desnecessários, busca de ajuda); e (3) a satisfação ou insatisfação (efeito subjetivo) que ela possa trazer ao usuário.

Reiterando a importância da avaliação de usabilidade, cabe salientar a visão de Cybis et al. (2010), estes afirmam que essa tem como intuito identificar problemas que possam vir a comprometer a interação do usuário com a interface. Este tipo de avaliação ganha ainda mais importância na análise em contexto educacional, uma vez que a baixa usabilidade dos sistemas, além de ser responsável pela frustração e diminuição de produtividade, pode levar o aluno a conclusões equivocadas ou até mesmo causar desinteresse pelo estudo de determinado conteúdo. As atividades ligadas a avaliação de usabilidade visam identificar, seja por prospecção, diagnóstico ou observação (que são os principais tipos de avalições disponíveis na literatura), os problemas de usabilidade em interfaces humano-computador e contribuir para a sua eliminação. Para realização da avaliação de usabilidade proposta neste trabalho foi utilizado o instrumento ErgoList desenvolvido no LabiUtil (CYBIS, 1997; CYBIS et al., 2000), o qual propõe a realização de uma sequência estruturada de avaliação de usabilidade, e possui um instrumento predefinido para sua execução.

Considerando esse instrumento cabe citar alguns dos principais objetivos de uma avaliação de usabilidade de acordo com alguns de seus idealizadores (CYBIS et al., 2000): (1) Constatar, observar e registrar, problemas efetivos de usabilidade durante a interação; (2) Calcular métricas objetivas sobre a eficácia, eficiência e produtividade do usuário na interação com o sistema; (3) Diagnosticar as características do projeto que provavelmente atrapalhem a interação, por estarem em desconformidade com padrões implícitos e explícitos de usabilidade; (4) Prever dificuldades de aprendizado na operação do sistema; (5) Prever os tempos de execução de tarefas informatizadas; 
(6) Conhecer a opinião do usuário em relação ao sistema; (7) Sugerir as ações de reprojeto mais evidentes, face os problemas de interação efetivos ou diagnosticados. Neste estudo o enfoque está no objetivo 1.

\section{METODOLOGIA}

Este estudo apresenta como objetivo central avaliar a usabilidade de dois OAs sobre o conteúdo de genética, a partir da perspectiva do usuário. Nesse sentido, destaca-se que do ponto de vista da abordagem do problema essa pesquisa é qualitativa, e a variável observada é a satisfação dos usuários ao utilizar os objetos de aprendizagem listados na seção 4. Quanto aos procedimentos metodológicos conduzidos na realização deste estudo, estes foram desenvolvidos basicamente em duas etapas: Na primeira etapa foi elaborada uma pesquisa bibliográfica para o levantamento de conceitos básicos inerentes ao tema em estudo; e Na segunda etapa foi realizada a avaliação da usabilidade dos OAs, por meio da aplicação de um instrumento de pesquisa, no formato de questionário (ErgoList), para possíveis usuários dos softwares, o que se caracteriza como uma avaliação prospectiva.

$\mathrm{Na}$ revisão literatura desenvolvida foram investigados conceitos de genética, informática em educação, usabilidade em objetos de aprendizagem digitais, os critérios de usabilidade que estes devem possuir para atender às necessidades dos usuários, bem como foi possível pesquisar sobre as formas de se avaliar um software educativo, e instrumentos de avaliação amplamente utilizados. Conforme Salomon (2004) uma pesquisa bibliográfica busca a resolução de um problema (hipóteses), por meio de referenciais teóricos publicados, analisando e discutindo as várias contribuições científicas. Esse tipo de pesquisa traz subsídios e conhecimento sobre o que está sendo pesquisado, como e sob que enfoque e/ou perspectivas foi tratado o assunto apresentado na literatura científica.

Quanto a segunda etapa, da avaliação dos OAs, inúmeros são os métodos de avaliação existentes. $\mathrm{Na}$ área educacional, nota-se a relevância desse processo, principalmente em projetos que têm por objetivo utilizar softwares educacionais, neste estudo configurados como Objetos de Aprendizagem, que contemplem as disciplinas escolares e contribuam para construção e fixação do conhecimento. Segundo Cybis (2003), os métodos de avaliação são divididos em três subcategorias, avaliação preditivo analítica, objetivo-empírica e prospectiva: (1) Preditiva/analítica - técnica que não necessita da participação do usuário, não é exigido o uso do software em uma situação real; (2) Objetivas/empíricas - o usuário tem participação ativa, com sessões de observação da interação, a exemplo da técnica de ensaios de interação; e (3) Prospectivas - caracterizam-se principalmente por exigir a presença do usuário, emitindo suas contribuições com suas experiências, opiniões e preferências, baseiam-se nas aplicações de questionários que relatam a satisfação ou insatisfação ao utilizarem o software.

Como já salientado, o método adotado para a avaliação dos OAs empregado neste estudo foi o de prospecção, avaliação de software em que a presença do usuário é fundamental, por meio dela é possível perceber, com base em quem realmente se beneficiará do instrumento, se esse tem pontos falhos ou não. Essa forma de avaliação é apoiada principalmente por aplicações de questionários aos usuários e na percepção de sua satisfação ou insatisfação ao utilizar o software. Dessa forma, para apoiar a avaliação conduzida no decorrer dessa pesquisa foi utilizado o instrumento de pesquisa ErgoList do LabiUtil (CYBIS, 1997; CYBIS et al., 2000; CYBIS et al., 2010), com sua aplicação foi possível verificar se os OAs avaliados atendem aos critérios de usabilidade vinculados a cada uma 
das questões realizadas. A descrição detalhada deste instrumento é apresentada na sequência (6.1), e o procedimento de aplicação na subseção seguinte (6.2).

\subsection{Instrumento de Avaliação: ErgoList - LabiUtil}

O questionário utilizado no decorrer deste processo de avaliação foi o ErgoList, baseado no conceituado trabalho de usabilidade do LabiUtil da Universidade Federal de Santa Catarina (CYBIS, 1997; CYBIS et al., 2000). Este instrumento é um sistema de avaliação de qualidade ergonômica de software composto de um checklist sobre as quais o usuário (avaliador) pode apor opiniões e observações na medida em que vai procedendo a avaliação. Cada uma de suas questões é associada a um critério de usabilidade que é avaliado pelo usuário, de forma simples e significativa. O Quadro 1 apresenta o questionário que foi utilizado no decorrer desta pesquisa, com as suas questões e o critério de usabilidade vinculado. Destaca-se que neste estudo não foram utilizadas todas as questões do ErgoList, apenas as consideradas mais relevantes para este estudo. As questões devem ser respondidas de forma afirmativa ou negativa apenas (sim/não).

Quadro 1 - Instrumento de Avaliação de Usabilidade ErgoList - LabiUtil

\begin{tabular}{|c|c|c|}
\hline $\mathbf{N}^{\circ}$ & QUESTÃO & CRITÉRIO \\
\hline 1. & $\begin{array}{l}\text { Verifique se existem descrições textuais associadas a imagens, gráficos, } \\
\text { sons, animações, ícones, vídeos. }\end{array}$ & Compatibilidade \\
\hline 2. & Verifique se o texto está escrito na voz ativa. & Compatibilidade \\
\hline 3. & $\begin{array}{l}\text { Verifique se o texto está escrito em linguagem simples, clara, familiar, no } \\
\text { idioma do usuário. }\end{array}$ & Compatibilidade \\
\hline 4. & $\begin{array}{l}\text { Verifique se o cursor é destacado nas telas, assumindo formatos e tamanhos } \\
\text { que permitam com que seja encontrado facilmente na tela por usuários de } \\
\text { diferentes idades. }\end{array}$ & Compatibilidade \\
\hline 5. & $\begin{array}{l}\text { Verifique se os componentes do site podem ser operados por meio de } \\
\text { diferentes dispositivos, em particular, teclado e mouse. }\end{array}$ & Flexibilidade \\
\hline 6. & $\begin{array}{l}\text { Verifique se há um contraste favorável entre as cores do texto e as do fundo } \\
\text { no qual o texto se encontra. }\end{array}$ & Legibilidade \\
\hline 7. & $\begin{array}{l}\text { Verifique se a informação expressa (decodificada) por cores (exemplo: cor + } \\
\text { sublinhado, cor + espessura de linha, etc.). }\end{array}$ & Legibilidade \\
\hline 8. & $\begin{array}{l}\text { Discriminação entre cores de mesmo matiz (tom), principalmente para azul, } \\
\text { verde e amarelo são difíceis de serem realizadas. }\end{array}$ & Legibilidade \\
\hline 9. & Verifique se o tamanho das fontes usadas nos textos é de mínimo 12 ou 14. & Legibilidade \\
\hline 10. & Verifique se os textos estão escritos em letras maiúsculas e minúsculas. & Legibilidade \\
\hline 11. & $\begin{array}{l}\text { Verifique se abreviaturas ou siglas encontram-se descritas (por extenso, em } \\
\text { legenda, etc.) }\end{array}$ & Legibilidade \\
\hline 12. & $\begin{array}{l}\text { Verifique se as páginas estão livres de atualizações periódicas automáticas } \\
\text { e, caso exista, se o usuário pode facilmente desativar este recurso. }\end{array}$ & $\begin{array}{l}\text { Controle do } \\
\text { usuário }\end{array}$ \\
\hline 13. & $\begin{array}{l}\text { Verifique se os objetos de controle, como links, botões de comando, caixas } \\
\text { de atribuição, barras de rolagem, etc. apresentam uma área visível às ações } \\
\text { dos usuários suficientemente grande para permitir um fácil e confortável } \\
\text { acionamento por parte dos usuários de diferentes idades. }\end{array}$ & $\begin{array}{l}\text { Agrupamento/ } \\
\text { distinção } \\
\text { por localização }\end{array}$ \\
\hline 14. & $\begin{array}{l}\text { Verifique se o destino de cada link está claramente identificado em seu } \\
\text { enunciado textual. }\end{array}$ & $\begin{array}{l}\text { Significado dos } \\
\text { códigos e } \\
\text { denominações }\end{array}$ \\
\hline 15. & $\begin{array}{l}\text { Em páginas com formulários, onde haja a entrada de dados e/ou de } \\
\text { comandos, verifique o correto posicionamento dos objetos de interação } \\
\text { (campo de adição, botão de rádio, lista de seleção, etc.) em relação aos seus } \\
\text { respectivos rótulos de identificação. }\end{array}$ & Presteza \\
\hline
\end{tabular}




\begin{tabular}{|c|l|c|}
\hline 16. & $\begin{array}{l}\text { Verifique se o usuário está livre de ações repetitivas durante a operação do } \\
\text { site. }\end{array}$ & Ações Mínimas \\
\hline 17. & $\begin{array}{l}\text { Verifique se informaç̃̃es e objetos de interação que ocorrem repetidos nas } \\
\text { diferentes páginas, são apresentados em posições consistentes. }\end{array}$ & Consistência \\
\hline 18. & $\begin{array}{l}\text { Verifique se as páginas estão livres de informações irrelevantes, repetitivas } \\
\text { ou impertinentes. }\end{array}$ & $\begin{array}{c}\text { Densidade } \\
\text { informacional }\end{array}$ \\
\hline
\end{tabular}

Fonte: LabiUtil ${ }^{7}$

\subsection{Aplicação do Questionário}

O questionário para avaliar a usabilidade dos OAs e o Termo de Consentimento Livre e Esclarecido (TCLE) foram enviados via e-mail sob forma de convite para participação nesta pesquisa, isso ocorreu no primeiro semestre do ano letivo de 2020. Foram convidados nove professores de Biologia, formados em Ciências Biológicas - Licenciatura Plena, com conhecimento na área específica dos Objetos de Aprendizagem e com experiência em docência no Ensino Médio, todos trabalham na rede pública (estadual e federal) e/ou privada de ensino no estado do Rio Grande do Sul, Brasil.

Juntamente com o questionário de usabilidade, também foi encaminhada a explicação geral e objetivos dos dois objetos de aprendizagem da área de genética, disponíveis no NOAS, sob os títulos: Montando Idiogramas e Síntese Proteica, bem como o link, descrição e instruções de uso. Os professores deveriam usar para si e não os aplicar nas turmas em que lecionam. Ainda, os participantes estavam livres para não responder o questionário, caso não se sentissem confortáveis a fazê-lo.

\section{RESULTADOS E DISCUSSÃO}

Após, aproximadamente um mês do envio dos questionários para os docentes, três destes retornaram, inclusive com ponderações. Dois não deram resposta, e outros 4 professores responderam o e-mail, mas alegaram não ter tempo para participar da pesquisa. Como já mencionado no decorrer do manuscrito, a avaliação dos objetos de aprendizagem se deu por prospecção e foram analisadas 18 perguntas, divididas em 10 critérios de usabilidade conforme o instrumento ErgoList do LabiUtil (seção 6.1) e representados nas Figuras 1 e 2.

A Figura 1 apresenta o gráfico com os critérios avaliados do OA "Montando Idiogramas", que como visto não atende alguns critérios em totalidade, como: agrupamento/distinção por localização, controle do usuário e flexibilidade (não permite que o usuário personalize a interface). Porém, isso não afetaria na utilização do objeto pelo aluno, porque parcialmente ainda está coerente com esses requisitos, o qual pode ainda auxiliar na aprendizagem. Contudo, uma ausência completa afetaria sua utilização, e recomendações de adequação deveriam ser realizadas. Ainda, seu uso foi indicado pelos professores participantes da pesquisa, pois outros critérios como significado dos códigos, o qual diz respeito a adequação entre o objeto ou a informação apresentada e sua referência, compatibilidade e legibilidade foram contemplados. Caso esse fosse utilizado em uma aula de genética, os discentes poderiam simular situações reais, de forma virtual fazendo com que esses alunos pudessem compreender de modo prático sobre cariótipos (conjunto de cromossomos de uma espécie) normais

\footnotetext{
${ }^{7}$ Disponível em: http://www.labiutil.inf.ufsc.br/
} 
ou com síndromes, o que talvez não seria possível apenas com uma aula expositiva e/ou utilizando um livro didático.

Figura 1 - Critérios atendidos ou não, após uso do Objeto de Aprendizagem - Montando Idiogramas

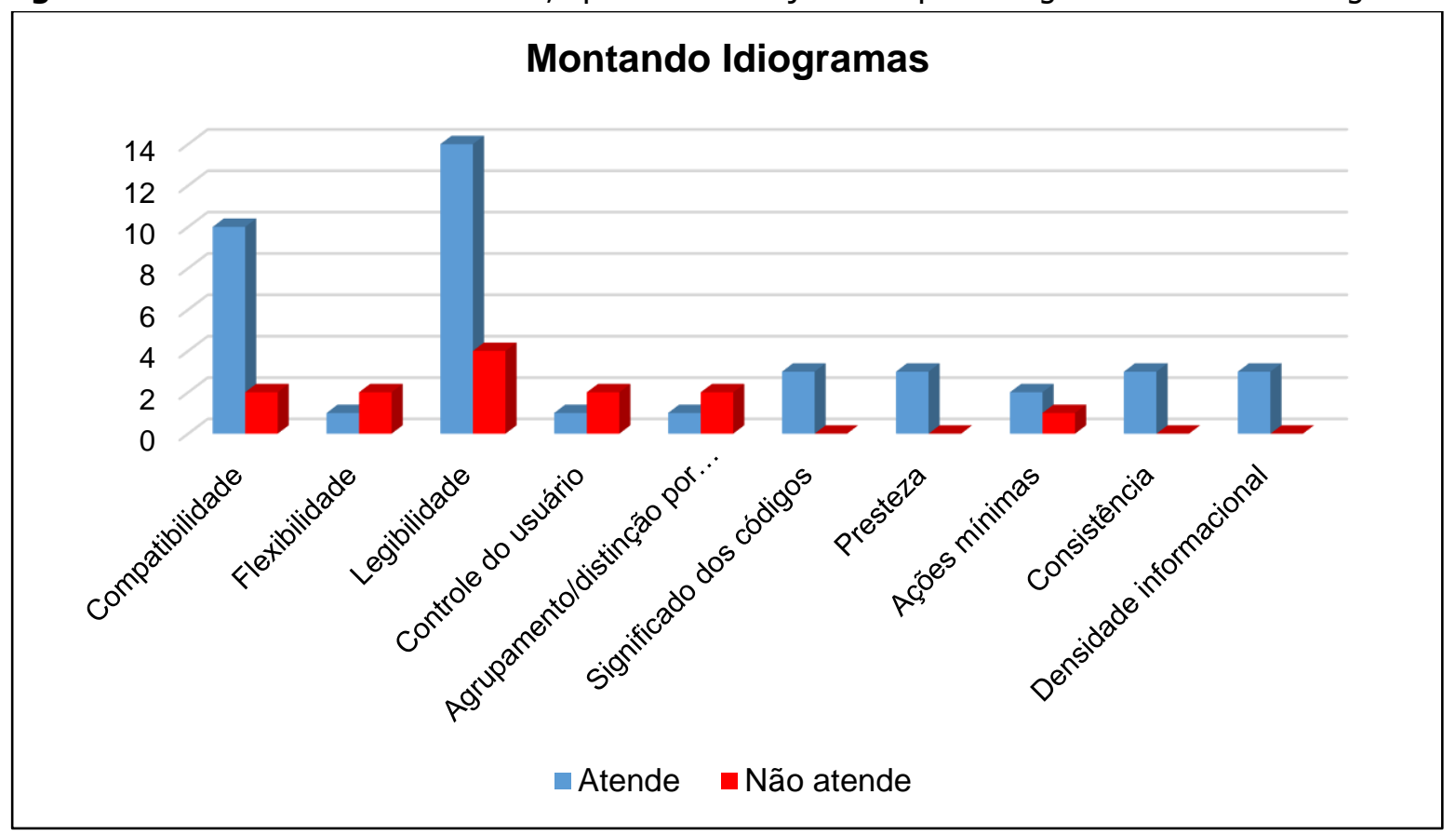

Fonte: Autoras (2021).

A Figura 2 faz menção ao gráfico de avaliação da usabilidade do OA "Síntese Proteica", que trata do fenômeno de síntese de proteínas, bastante complexo e essencial aos seres vivos. O conteúdo abordado neste objeto é de difícil compreensão para alunos, e por vezes moroso de se trabalhar em sala de aula, devido a abstração de conceitos não contemplados apenas com a aula expositiva e/ou apenas usando o livro didático. Analisando a Figura 2, pode-se observar que 3 dos 10 critérios atenderam satisfatoriamente os docentes, os outros atenderam parcialmente suas expectativas.

Figura 2 - Critérios atendidos ou não, após uso do Objeto de Aprendizagem - Síntese Proteica.

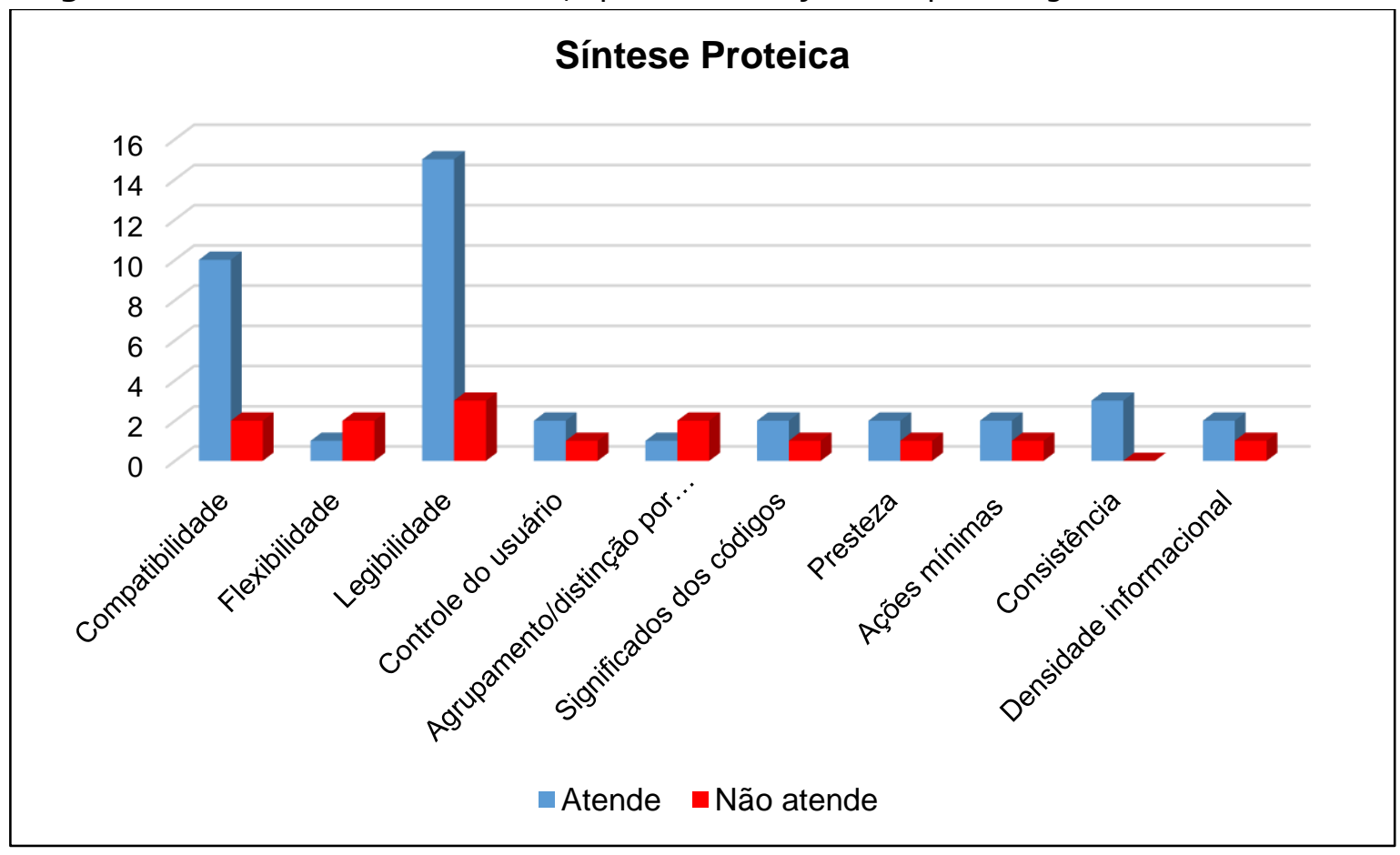

Fonte: Autoras (2021). 
Ao fim do questionário foi realizada uma pergunta aberta para os docentes, por meio das respostas foi possível perceber a aceitação dos professores quanto a utilização destes softwares em suas aulas, a questão e as respostas podem ser observadas no Quadro 2.

Quadro 2 - Instrumento de Avaliação de Usabilidade LabiUtil

\begin{tabular}{|c|c|}
\hline QUESTÃO & Respostas \\
\hline \multirow{3}{*}{$\begin{array}{l}\text { Você usaria estes objetos de } \\
\text { aprendizagem em suas aulas? } \\
\text { Justifique. }\end{array}$} & $\begin{array}{l}\text { Professor } 1 \text { - "Sim. Pois trazem uma abordagem mais atraente para } \\
\text { os estudantes e, além disso, permitem que os mesmos se tornem } \\
\text { agentes de seu aprendizado, os objetos educacionais são formas } \\
\text { complementares de abordar temas abstratos, como Genética, } \\
\text { mesmo em escolas sem a estrutura de um laboratório de Biologia". }\end{array}$ \\
\hline & $\begin{array}{l}\text { Professor } 2 \text { - "Sim, pois permitem dar concretude a conceitos } \\
\text { abstratos ou de difícil visualização na prática, além da possibilidade } \\
\text { de interação dos alunos com o objeto de aprendizagem". }\end{array}$ \\
\hline & $\begin{array}{l}\text { Professor } 3 \text { - "Sim, porque muitas vezes não há disponibilidade de } \\
\text { observação de certos fenômenos, em especial em escolas que não } \\
\text { contam com laboratórios, dessa forma com recursos como estes, é } \\
\text { possível mostrar aos alunos como alguns eventos acontecem, o que } \\
\text { completa o processo de ensino e aprendizagem". }\end{array}$ \\
\hline
\end{tabular}

Fonte: Autoras (2021).

As respostas dos professores, condizem e são amparadas pelo pensamento de Moran, Masetto e Behrens (2006), em que os autores mencionam que as Tecnologias de Informação e Comunicação, nesse caso representadas pelos OAs, podem constituir-se como um elemento na valorização das práticas pedagógicas vivenciadas nas aulas de biologia/genética, uma vez que acrescenta maior dinâmica nos processos de ensino e aprendizagem, fornecendo maior acesso à informação e assim dando outra visão dos conteúdos trabalhados. Desta forma, a utilização de tecnologias na prática pedagógica oferece a possibilidade de diversificar as aulas, facilitando a apresentação e a explicação dos conteúdos, visto que dispõe de inúmeros recursos midiáticos como: imagens, vídeos, sons entre outros.

Quanto à avaliação de usabilidade dos OAs, observou-se que os critérios legibilidade (6 questões) e compatibilidade (4 questões) foram os melhores avaliados pelos professores, devido a facilidade de interação, o que instigou a concluir que utilização dos OAs pode ter impactos positivos. Já o critério de controle do usuário não atendeu satisfatoriamente as expectativas dos docentes, o que pode ser explicado pelo fato do usuário não conseguir interferir na dinâmica da interface, limitando-o a fazer apenas o que o software propõe. Além desse, o critério de agrupamento/distinção por localização não atendeu aos docentes de forma satisfatória, o que significa que objetos de controle, como links, botões de comando, caixas de atribuição, barras de rolagem, não apresentam uma área visível às ações dos usuários, que sejam suficientemente grandes para permitir um fácil e confortável acionamento por parte dos usuários de diferentes idades. Ainda, observou-se que o software precisa possuir uma boa usabilidade para que os usuários (aqui representados por professores) sintam-se motivados a utilizá-los.

Dessa forma, destaca-se a importância dos docentes se capacitarem para utilizar novos recursos em suas aulas, como os OAs descritos neste estudo. Recursos estes, que podem possibilitar aos alunos terem maior sucesso no processo de ensino e aprendizagem, sobretudo em conteúdos que são mais complexos, como no caso da genética. 


\section{CONSIDERAÇÕES FINAIS}

Os Objetos de Aprendizagem despontam como instrumentos positivos no que se refere a beneficiar professores e alunos como um recurso alternativo no processo de ensino e aprendizagem de modo eficiente, mesmo em locais com poucos recursos tecnológicos. Todavia, como qualquer produto de software nem todos os OAs que estão disponíveis para utilização cumprem com seus objetivos, qual seja: ensinar sobre determinado assunto/conteúdo de forma satisfatória. Por isso, avaliar de forma adequada um software educativo antes de sua aplicação deveria ser uma tarefa comum entre os docentes. Nesse sentido, o intuito desde estudo foi principalmente o de demostrar como é o processo de avaliação de um software educativo, com foco em analisar sua usabilidade.

Para isso, foi desenvolvida uma avaliação por prospecção que se caracteriza principalmente pela participação do usuário, o qual emite sua opinião com base na utilização do software, esse tipo de avaliação é realizado em especial com aplicação de instrumentos de pesquisa. O usuário foi representado neste estudo por professores experientes e graduados em biologia e o instrumento utilizado foi o questionário ErgoList do LabiUtil. Este instrumento é muito empregado em avaliações de usabilidade em softwares educacionais e é composto pelos seguintes critérios: (1) Compatibilidade, (2) Flexibilidade, (3) Legibilidade, (4) Controle do usuário, Agrupamento/distinção por localização, (6) Significado dos códigos e denominações, (7) Presteza, (8) Ações Mínimas, (9) Consistência e (10) Densidade informacional.

Os dois OAs avaliados foram: Montando Idiogramas e Síntese Proteica, disponíveis no repositório NOAS, com temática vinculada a genética. Os OAs foram melhores avaliados, nos critérios de legibilidade (6 questões) e compatibilidade (4 questões), enquanto que a pior avaliação ficou com os critérios de controle do usuário (1 questão) e agrupamento/distinção por localização (1 questão). Dessa forma, mesmo com esses dois últimos critérios não atendendo as expectativas, constatou-se que os OAs podem ser usados como facilitadores das aulas de biologia/genética para o Ensino Médio. Além disso, verificou-se que esses estão condizentes para utilização de alunos, ideia amparada nos resultados obtidos por meio de análise dos questionários de usabilidade respondidos pelos professores da área.

Para finalizar, destaca-se que a utilização de OAs pode trazer resultados positivos no processo de ensino e aprendizagem, por meio da ludicidade e interação humano/computador, as aulas tendem a ficar mais dinâmicas, motivadoras e significativas, podendo levar os alunos ao êxito tanto no que se refere a construção do conhecimento, gosto pelas ciências, aprovação escolar e em exames como o ENEM.

\section{REFERÊNCIAS}

ADOLFO, M.; MACHADO D.; WARPECHOWSKI, M. Ensino e Aprendizagem de Biologia no Ensino Médio através da Informática. Educativa VI Congresso Brasileiro de Informática na Educação (CBIE 2017), Anais do XXIII Workshop de Informática na Escola (WIE 2017), 2017.

ARELLANO, M. A. M. Repositórios digitais DSpace. 2008. Disponível em: <http://dspace.ibict.br/dmdocuments/RepositoriosInstitucionaisDSpace.pdf> Acesso em: 06 maio 2021. 
ABNT - ASSOCIAÇÃO BRASILEIRA DE NORMAS TÉCNICAS. NBR ISO 9241: Ergonomia da interação humano-sistema. Parte 151: Orientações para interfaces de usuários da World Wide Websistemas interativos. Rio de Janeiro: ABNT, 2011.

BARNI, G. S. A importância e o sentido de estudar genética para estudantes do terceiro ano do ensino médio em uma escola da rede estadual de ensino em Gaspar (SC). 2010. 184 f. Dissertação (Mestrado Ciências Naturais e Matemática)-Universidade Regional de Blumenau, Blumenau 2010.

BONZANINI, T. K.; BASTOS, F. Temas da Genética contemporânea e o Ensino de Ciências: que materiais são produzidos pelas pesquisas e que materiais os professores utilizam? In: VIII ENPEC I CIEC, Campinas/SP 2011. Disponível em:

<http://www.nutes.ufrj.br/abrapec/viiienpec/resumos/R0389-2.pdf.> Acesso em: 21 abr. 2021.

CARVALHO, A. A. A. Como olhar criticamente o software educativo multimídia. São Paulo: Abril, Editora, p. 200 - 215, 2005.

CYBIS, W. A. Abordagem ergonómica para IHC, Apostila LablUtil. Universidade Federal de Santa Catarina, 1997.

CYBIS, W. A.; PIMENTA, M. S.; SILVEIRA, M. C.; GAMEZ, L. Uma Abordagem Ergonômica para o Desenvolvimento de Sistemas Interativos. 2000. Disponível em:

<https://www.unicamp.br/ ihc99/Ihc99/AtasIHC99/AtasIHC98/Cybis.pdf>. Acesso em: 07 maio 2021.

CYBIS, W. A. Engenharia de Usabilidade: uma abordagem ergonômica. 2003. Apostila para o curso de Pós-Graduação em Engenharia de Produção - Laboratório de Utilizabilidade de Informática - Universidade de Santa Catarina: Florianópolis, 2003.

CYBIS, W. A.; BETIOL, A. H.; FAUST, R. Ergonomia e usabilidade: conhecimentos, métodos e aplicações. 2.ed. São Paulo: Novatec, 2010.

DAGNINO, R. P.; DIAS, R. A política de C\&T brasileira: três alternativas de explicação e orientação. Revista Brasileira de Inovação, Rio de Janeiro, v. 6, n. 2, p. 373-403, 2007.

GUEDES, F. D.; CASTRO FILHO, J. A. A seleção de objetos educacionais digitais por professores. In: Simpósio brasileiro de informática na educação SBIE. 2010. Disponível em: <http://www.br-ie.org/pub/index.php/sbie/article/view/1491/1256>. Acesso em: 03 ago. 2021.

JUSTINA, L. A. D. Ensino de Genética e história de conceitos relativos à hereditariedade. 2001, 145 f. Dissertação (Mestrado em Biologia)-Universidade Federal de Santa Catarina, Centro de Ciências da Educação, Florianópolis, 2001.

LABIUTIL. Laboratório de Utilizabilidade da Informática. Disponível em: <http://www.labiutil.inf.ufsc.br/>. Acesso em: 05 maio 2021.

MAGUIRE, M.; et al. A comparison of three materials used for tactile symbols to communicate colour to children and young people with visual impairments. British Journal of Visual Impairment, v. 34, n.1, p. 54-71, 2016.

MORAN, J. M; MASETTO, M. T.; BEHRENS, M. A. Novas tecnologias e mediação pedagógica. 1. ed. Campinas: Papirus, 2006. Disponível em:

<https://www.academia.edu/10222269/Moran_Masetto_e_Behrens_NOVAS_TECNOLOGIAS_E_ME DIA\%C3\%87AO_PEDAGOGICA>. Acesso em: 20 abr. 2021. 
MORELLATO, V.; et al. Softwares Educacionais e a Educação Especial: Refletindo sobre Aspectos Pedagógicos. Revista Novas Tecnologias na Educação, v. 4, n. 1, p.1-10, 2006.

MOURA J.; et al. Biologia/Genética: $O$ ensino de biologia, com enfoque a genética, das escolas públicas no Brasil - breve relato e reflexão. Ciências Biológicas e da Saúde, v. 34, n. 2, p. 167174, 2013.

NIELSEN, J. Usability Engineering. Moragan Kaufmann. 1. Ed. San Francisco: Morgam Kaufimann, Elsevier, 1993.

NIELSEN, J.; LORANGER, H. Usabilidade na Web. 1. ed. rev. e atual. Rio de Janeiro: Elsevier, 2007.

NOAS. Núcleo de Desenvolvimento de Objetos de Aprendizagem Significativa. Disponível em: <http://www.noas.com.br/>. Acesso em: 12 abr. 2021.

OLIVEIRA, C.; MOURA, S. P.; SOUSA, E. R. TIC's na educação: a utilização das tecnologias da informação e comunicação na aprendizagem do aluno. Pedagogia em Ação, v. 7 n. 1, p. 75-95, 2015.

ROCHA, da S. L. Estratégias metodológicas para ensinar genética no ensino médio. 2013. 47 f. Monografia (Especialização em Educação)-Universidade Tecnológica Federal do Paraná, Medianeira, 2013.

SALOMON, D. V. Como fazer uma monografia. 11. ed. São Paulo: Martins Fontes; 2004.

SANTOS, G. L (organizador) Tecnologias na Educação e Formação de Professores. Publicação Coletiva dos Professores Pesquisadores da área de Tecnologias na Educação do Programa de PósGraduação em Educação da Faculdade de Educação da Universidade de Brasília. Brasília: Plano, 2003.

SCAPIN, D. L.; BASTIEN, J. M. C. Ergonomic Criteria for the Evaluation of Human-Computer Interfaces. Relatório Técnico No 156 - Institut National de Recherche en Informatique et en Automatique (INRIA), França, 1993. Disponível em: <http://hal.inria.fr/docs/00/07/00/12/PDF/RT0156.pdf>. Acessado em: 16 set. 2021.

SILVA, N. L. R.; FERNANDES, E. P. B.; SOBREIRA, M. A genética no ensino médio: analisando uma visão discente. In: V CONEDU. Olinda/PE, v. 1, 2018. Disponível em:

<http://www.editorarealize.com.br/revistas/conedu/anais.php>. Acesso em: 28 abr. 2021.

SOUZA, de M. Dificuldades de alunos do ensino médio na aprendizagem de genética.

UFPR. Foz do Iguaçu, 2015. Disponível em:

<https://acervodigital.ufpr.br/bitstream/handle/1884/42247/R\%20-\%20E\%20-

\%20MARINEZ\%20DE\%20SOUZA.pdf?sequence=1\&isAllowed=y>. Acesso em: 03 ago. 2021.

SOUZA, M. G. $O$ uso da internet como ferramenta pedagógica para os professores do ensino fundamental. 2013. 59 f. Monografia (Graduação em Licenciatura Plena em Informática)Universidade Aberta do Brasil, Universidade Estadual do Ceará, Centro de Ciências e Tecnologia, Curso de Licenciatura Plena em Informática, Tauá/CE. 2013.

TAROUCO, L. M. R.; et al. Objetos de Aprendizagem. Teoria e Prática - CINTED/UFRGS. Porto Alegre: Evangraf, 2014.

TAVARES N. R. B. História da informática educacional no Brasil observada a partir de três projetos públicos. São Paulo: Escola do Futuro, 2002. 
VALENTE, J. A. E.; ALMEIDA, F. J. Visão analítica da informática na educação no Brasil: A questão da formação do professor. Revista Brasileira de Informática na Educação, v. 1, n. 1, p. 1-28, 1997.

WILEY, D. A. Connecting learning objects to instrucional design theory: A definition, a metaphor, and a taxonomy. The instrucional use of learning objects, 2002. Disponível em: <http://wesrac.usc.edu/wired/bldg-7_file/wiley.pdf/wiley.pdf> Acesso em: 08 ago. 2021.

Submissão: 15/07/2021

Aceito: 18/09/2021 